\title{
Intrinsic Fermi-surface contribution to the bulk photovoltaic effect
}

\author{
Lingyuan Gao $\odot,{ }^{1, *}$ Zachariah Addison $\odot,{ }^{2, *}$ E. J. Mele, ${ }^{2}$ and Andrew M. Rappe ${ }^{1, \dagger}$ \\ ${ }^{1}$ Department of Chemistry, University of Pennsylvania, Philadelphia, PA 19104-6323, USA \\ ${ }^{2}$ Department of Physics and Astronomy, University of Pennsylvania, Philadelphia, PA 19104, USA
}

(Received 19 November 2020; revised 18 October 2021; accepted 21 October 2021; published 29 November 2021; corrected 10 December 2021)

\begin{abstract}
We study the Fermi surface contribution to the nonlinear DC photocurrent at quadratic order in a spatially uniform optical field in the ultraclean limit. In addition to shift and injection current, we find that polarized light incident on a metallic system generates an intrinsic contribution to the bulk photovoltaic effect deriving from photoinduced electronic transitions on the Fermi surface. In velocity gauge, this contribution originates in both the coherent band off-diagonal and diagonal parts of the density matrix, describing, respectively, the coherent wave function evolution and the carrier dynamics of an excited population. We derive a formula for the intrinsic Fermi surface contribution for a time-reversal invariant chiral Weyl semimetal illuminated with circularly polarized light. At low frequency, this response is proportional to the frequency of the driving field, with its sign determined by the topological charge of the Weyl nodes and with its magnitude being comparable to the recently discovered quantized circular photogalvanic effect. Our work presents a complete derivation for all contributions to nonlinear DC photocurrent and classifies them according to the polarization of light in the presence and absence of time-reversal symmetry.
\end{abstract}

DOI: 10.1103/PhysRevResearch.3.L042032

\section{INTRODUCTION}

Interest in developing new platforms for efficient solar energy conversion has drawn attention to the photovoltaic properties of new materials and the physics of their lightmatter interactions. The bulk photovoltaic effect (BPVE), sometimes also referred to as the photogalvanic effect (PGE), has attracted much attention, as it can directly convert light to a DC current [1]. The BPVE is a second-order nonlinear response that can be decomposed into terms that are symmetric and antisymmetric in the polarization states of the light, corresponding to a linear photogalvanic effect (LPGE) and a circular photogalvanic effect (CPGE), respectively [1]. The ballistic current is an important mechanism of the BPVE and emerges in both LPGE and CPGE [2-5], where an asymmetric distribution of photoexcited charge carriers on the conduction band is induced by electron-phonon or electron-electron scattering. Apart from the ballistic current, the CPGE is usually described as an injection current. Owing to the phase lag between orthogonal components of a circularly polarized beam, an asymmetry in the excited state population at time-reversed momenta $\mathbf{k}$ and $-\mathbf{k}$ can be induced [6,7]. In a two-band model, the carrier generation rate can be related to the Berry curvature, which relates the trace of the CPGE tensor to the quantized topological charge of degenerate points

\footnotetext{
*These authors contributed equally to this work.

Corresponding author: rappe@ sas.upenn.edu
}

Published by the American Physical Society under the terms of the Creative Commons Attribution 4.0 International license. Further distribution of this work must maintain attribution to the author(s) and the published article's title, journal citation, and DOI. in the band structure for a Weyl semimetal [8]. In LPGE, other than ballistic current, shift current can be described as a coherent response associated with the real-space shift of an electron induced by a dipole-mediated vertical inter-band transition [9]. Recent photo-Hall measurements of both LPGE and CPGE in an applied magnetic field have successfully separated the shift and ballistic contributions to the electric charge current $[10,11]$. First principles studies [12-14] of the shift current have led to the prediction and discovery of many new photovoltaic materials [15-21].

Light-matter interactions in these materials are described by coupling electrons in the material to the electromagnetic potentials $A_{\mu}$. In the velocity gauge, the external electric potential is taken to vanish, and the external electromagnetic vector potential can be incorporated into the electronic Hamiltonian through a minimal coupling procedure that augments the electronic momentum operator $\hat{\boldsymbol{p}} \rightarrow \hat{\boldsymbol{p}}+$ $\boldsymbol{e A}(\boldsymbol{r}, t) / \hbar[22,23]$. For a spatially uniform, but time varying electric field and a vanishing magnetic field, a time-dependent gauge transformation on the electronic wave function can bring the effective Hamiltonian back to its original unperturbed form with the addition of an electric-field-induced perturbation $\delta H=e \boldsymbol{E}(t) \cdot \hat{\boldsymbol{r}}$ [24,25]. This form of the Hamiltonian is called the length gauge and has been used to derive expressions for the contributions to nonlinear electric currents in insulators and semimetals [6,26,27].

Using time-dependent perturbation theory in velocity gauge, we derive formulas for the nonlinear DC photocurrent induced at quadratic order in an external driving field. We find a geometric contribution to the second-order current related to the curvature of the electronic Bloch bands that must vanish for insulating materials, but can be nonzero for metals and semimetals. This contribution to the current derives from both 
off-diagonal and time-independent band diagonal contributions in the density matrix, and it is proportional to $\boldsymbol{k}$-space derivatives of the unperturbed Fermi occupation factors multiplied by the band-resolved Berry curvature or quantum metric tensor [28,29], depending on the polarization of the light. For a time-reversal (TR) invariant system, the current is only non-vanishing under circularly polarized (CP) illumination. By breaking time-reversal symmetry (TRS), both circularly and linearly polarized light can induce a current. At zero temperature, the current derives from dipole-allowed vertical interband transitions for electrons whose crystal momenta are near the Fermi surface. Thus we name this photocurrent the "intrinsic Fermi surface contribution", defining an additional contribution to the CPGE and LPGE responses.

As an example, we calculate this contribution to the CPGE in a three-dimensional time-reversal symmetric, but inversion symmetry broken Weyl semimetal. We show that for an isotropic Weyl node, this response is proportional to the Weyl node's charge weighted by a factor that depends on the energy of the Bloch bands near the Fermi surface. In the small $\omega$ limit, the photovoltaic response for an isolated Weyl node is purely quantized and would therefore directly measure the topological charge of the Weyl point. When mirror symmetries are broken in a chiral Weyl semimetal, point nodes with opposite topological charges are offset in energy, allowing a nonzero DC charge current to flow. This can be distinguished from the extrinsic quantized circular photogalvanic effect deriving from the injection contribution to the current, which is proportional to a scattering time $\tau[8]$.

\section{GENERAL THEORY}

We study the Hamiltonian for a particle with charge $-e$ coupled to a time-dependent vector potential $\boldsymbol{A}(t)$

$$
\hat{H}(\hat{\boldsymbol{r}}, \hat{\boldsymbol{p}}, t)=\frac{(\hat{\boldsymbol{p}}+e \boldsymbol{A}(t))^{2}}{2 m_{e}}-e V(\hat{\boldsymbol{r}}),
$$

where $V(\hat{\boldsymbol{r}})$ is the crystal potential, $m_{e}$ is the mass of the electron, and $\hat{\boldsymbol{p}}$ and $\hat{\boldsymbol{r}}$ are the electronic momentum and position operators. We work in velocity gauge, where the response to the electric field is made by the identification $\boldsymbol{E}(t)=-\partial_{t} \boldsymbol{A}(t)$. Here, we focus on the single-particle Hamiltonian, $\hat{H}(\hat{\boldsymbol{r}}, \hat{\boldsymbol{p}}, t)$ and do not include the contributions deriving from many-body interactions such as excitonic effects in our model. The nonlinear DC charge current is determined by calculating the trace of the product of the velocity operator $\hat{\boldsymbol{v}}(t)=i[\hat{H}(\hat{\boldsymbol{r}}, \hat{\boldsymbol{p}}, t), \hat{\boldsymbol{r}}] / \hbar$ and density matrix $\hat{\rho}(t)$. To isolate the nonlinear response, we expand both $\hat{\boldsymbol{v}}(t)$ and $\hat{\rho}(t)$ in a power series of $\boldsymbol{A}(t)$ up to quadratic order in the external driving field (denoted by the superscripts on the operators below) and calculate the current as

$$
\boldsymbol{j}(t)=\frac{1}{V} \sum_{n=0}^{2} \operatorname{Tr}\left[e \hat{\boldsymbol{v}}^{(n)}(t) \hat{\rho}^{(2-n)}(t)\right]
$$

(See Supplemental Material [30] for details). To solve for $\hat{\rho}(t)$, we employ the von Neumann equation which describes the time evolution of this quantum operator [31]:

$$
i \hbar \frac{d \hat{\rho}(t)}{d t}=[\hat{H}(\hat{\boldsymbol{r}}, \hat{\boldsymbol{p}}, t), \hat{\rho}(t)] .
$$

The Hamiltonian $\hat{H}(\hat{\boldsymbol{r}}, \hat{\boldsymbol{p}}, t)$ can be divided into two pieces. $\hat{H}_{0}(\hat{\boldsymbol{r}}, \hat{\boldsymbol{p}})=\frac{\boldsymbol{p}^{2}}{2 m_{e}}+V(\hat{\boldsymbol{r}})$ describes the unperturbed Hamiltonian before application of the external field and $\hat{H}^{\prime}(\hat{\boldsymbol{r}}, \hat{\boldsymbol{p}}, t)=$ $\frac{e \boldsymbol{A}(t) \cdot \hat{\boldsymbol{p}}}{m_{e}}+\frac{e^{2} \boldsymbol{A}(t) \cdot \boldsymbol{A}(t)}{2 m_{e}}$ describes the interaction between electrons in the material and the external field. With this substitution, we can solve Eq. 3 for the density matrix order by order in the vector potential $\boldsymbol{A}(t)$. Here, for simplicity Eq. (1) starts from a non-relativistic quadratic Hamiltonian. In Supplemental Material, we show an equivalent derivation for a general Bloch Hamiltonian $\hat{H}(\mathbf{k})$.

We focus on external driving fields with few nonzero Fourier components and write $\boldsymbol{A}(t)=\sum_{\omega^{\prime}} \boldsymbol{A}\left(\omega^{\prime}\right) e^{i\left(\omega^{\prime}-i \eta\right) t}$ with $\boldsymbol{A}\left(\omega^{\prime}\right)=\boldsymbol{A}^{*}\left(-\omega^{\prime}\right)$. The limit as $\eta \rightarrow 0$ denotes an adiabatic turning on of the electromagnetic field. At second order in the electromagnetic vector potential, the current couples to two different electromagnetic fields with unique Fourier components at two general frequencies $\omega_{1}$ and $\omega_{2}$. The DC limit is found by expanding the current in powers of $\omega_{1}+\omega_{2}$ to extract divergent and finite contributions to the current as $\omega_{1} \rightarrow-\omega_{2}$ and $\eta \rightarrow 0$.

\section{FERMI SURFACE CONTRIBUTION}

We parse the nonlinear DC photocurrent into its diagonal $\boldsymbol{j}^{\text {dia }} \sim \sum e \boldsymbol{v}_{n n}(\boldsymbol{k}) \rho_{n n}(\boldsymbol{k})$ and off-diagonal $\boldsymbol{j}^{\text {off }} \sim \sum_{n \neq m} e \boldsymbol{v}_{n m}$ $(\boldsymbol{k}) \rho_{m n}(\boldsymbol{k})$ parts. Here $\mathcal{O}_{n m}(\boldsymbol{k})=\left\langle\Psi_{n}(\boldsymbol{k})|\hat{\mathcal{O}}| \Psi_{m}(\boldsymbol{k})\right\rangle$, where $\left|\Psi_{n}(\boldsymbol{k})\right\rangle$ are the Bloch eigenstates of the unperturbed Hamiltonian $\hat{H}_{0}(\hat{\boldsymbol{r}}, \hat{\boldsymbol{p}}) \cdot \boldsymbol{j}^{\text {dia }}$ describes the current generated from the dynamics of excited carrier populations: terms in $j^{\text {dia }}$ are proportional to both the velocity, $\boldsymbol{v}_{n n}(\boldsymbol{k})$, and population density, $\rho_{n n}(\boldsymbol{k})$, of Bloch electrons in bands $n$ with crystal momentum $\boldsymbol{k}$. As shown by Eqs. (S20)-(S23) in Supplemental Material, $j^{\text {dia }}$ can be broken into three pieces but belonging to two types: (I) the divergent terms $\boldsymbol{j}^{\text {dia1 }}$ and $\boldsymbol{j}^{\text {dia2 }}$ proportional to $1 / \eta e^{2 \eta t}$ that diverge as $\eta \rightarrow 0$ and (II) $j^{\text {dia3 }}$ terms that are finite as $\eta \rightarrow 0$. However, the generation rate $\partial_{t} j^{\text {dial }}$ remains finite. This is called injection current $j^{\text {inj }}$, induced by resonant excitations between Bloch electrons in different bands with the same crystal momentum whose energy differs by $\hbar \omega$.

The non-divergent diagonal contribution, $\boldsymbol{j}^{\mathrm{dia} 3}$, can be written as

$$
\begin{aligned}
\boldsymbol{j}^{\mathrm{dia} 3}= & \frac{e^{3}}{2 V \hbar} \sum_{n, m, i, j, \boldsymbol{k}, \omega^{\prime}= \pm \omega}\left(f_{n}^{T}(\boldsymbol{k}, \mu)-f_{m}^{T}(\boldsymbol{k}, \mu)\right) \\
& \times \nabla_{\boldsymbol{k}}\left(\frac{1}{\varepsilon_{n}(\boldsymbol{k})-\varepsilon_{m}(\boldsymbol{k})+\hbar \omega^{\prime}}\right) \\
& \times v_{n m}^{i}(\boldsymbol{k}) v_{m n}^{j}(\boldsymbol{k}) A^{i}\left(\omega^{\prime}\right) A^{j}\left(-\omega^{\prime}\right) .
\end{aligned}
$$

Here, $\mu$ is the electron chemical potential. We note that $j^{\text {dia3 }}$ is rarely discussed in previous studies, but is an intrinsic contribution to the induced photocurrent: different from $\boldsymbol{j}^{\text {dial }}$ and $j^{\mathrm{dia} 2}$, it is not proportional to $1 / \eta$.

The contribution $\boldsymbol{j}^{\text {off }}$ describes a current generated from the coherence between electronic Bloch states in different bands. At second order in the perturbing field, a third band state $l$ is inserted as an intermediate transition state between the coherent pair $n$ and $m$. As shown in Supplemental Material [Eqs. (S24),(S25)], the three-band processes $n \rightarrow l \rightarrow m$ can 
be converted to an effective $n \leftrightarrow m$ interband transition with an energy factor $\frac{1}{\varepsilon_{n}(\boldsymbol{k})-\varepsilon_{m}(\boldsymbol{k})+\hbar \omega^{\prime}-i \hbar \eta}$. This can be decomposed into a resonant contribution to the current, $\boldsymbol{j}^{\text {off1 }}$, proportional to a $\delta$ functions and an off-resonant contribution to the current, $j^{\text {off2 }}$, proportional to the principle part of the quotient, representing two type of couplings. $\boldsymbol{j}^{\text {off1 }}$ is associated with the shift current $j^{\text {shift }}$, where the electron coordinate in real space is shifted along with the resonant excitation from band $n$ to $m$. For $j^{\text {off2 }}$, after removing terms that vanish identically, it can be written as

$$
\begin{aligned}
\boldsymbol{j}^{\mathrm{off} 2}= & \frac{e^{3}}{2 V \hbar} \sum_{n, m, i, j, \boldsymbol{k}, \omega^{\prime}= \pm \omega}\left(f_{n}^{T}(\boldsymbol{k}, \mu)-f_{m}^{T}(\boldsymbol{k}, \mu)\right) \\
& \times \frac{1}{\varepsilon_{n}(\boldsymbol{k})-\varepsilon_{m}(\boldsymbol{k})+\hbar \omega^{\prime}} \\
& \times \nabla_{\boldsymbol{k}}\left(v_{n m}^{i}(\boldsymbol{k}) v_{m n}^{j}(\boldsymbol{k})\right) A^{i}\left(\omega^{\prime}\right) A^{j}\left(-\omega^{\prime}\right) .
\end{aligned}
$$

We note that for insulators with a minimum band gap energy, $E_{\text {gap }}$, terms contributing to both $\boldsymbol{j}^{\text {off2 }}$ and $\boldsymbol{j}^{\text {dia3 }}$ involve fieldinduced dipole mediated transitions between Bloch states that lead to nonzero currents even for light with frequency $|\hbar \omega|<$ $E_{\text {gap }}$.

Though $\boldsymbol{j}^{\text {off2 }}$ and $\boldsymbol{j}^{\text {dia3 }}$ derive from different light-induced transition processes between Bloch states, the sum of the two contributions to the current can be simplified into a single term proportional to $\boldsymbol{k}$-space derivatives of the unperturbed Fermi occupation factors $f_{n}^{T}(\boldsymbol{k}, \mu)$ :

$$
\begin{aligned}
\boldsymbol{j}^{\mathrm{off} 2}+\boldsymbol{j}^{\mathrm{dia} 3}= & -\sum_{n, m, i, j, \boldsymbol{k}, \omega^{\prime}= \pm \omega} \frac{e^{3}}{V \hbar} \nabla_{\boldsymbol{k}} f_{n}^{T}(\boldsymbol{k}, \mu) \\
& \times \frac{v_{n m}^{i}(\boldsymbol{k}) v_{m n}^{j}(\boldsymbol{k})}{\varepsilon_{n}(\boldsymbol{k})-\varepsilon_{m}(\boldsymbol{k})+\hbar \omega^{\prime}} A^{i}\left(\omega^{\prime}\right) A^{j}\left(-\omega^{\prime}\right) .
\end{aligned}
$$

At zero temperature, $\boldsymbol{k}$-space derivatives of the Fermi occupation functions are proportional to a $\delta$ function that is nonzero only for crystal momenta along the Fermi surface. This important simplification clarifies the absence of DC current generation for insulators perturbed by light with frequency $|\hbar \omega|<E_{\text {gap }}$. In experiment, only the total current is measured, and these sub-gap currents arising from $j^{\text {off2 }}$ and $\boldsymbol{j}^{\text {dia3 }}$ cancel, leaving only contributions to the current for materials with non-vanishing Fermi surfaces. Therefore, this contribution is nonzero only for metallic systems; we denote this contribution to the current in Eq. 6 as $j^{\text {Fermi }}$. At finite temperature, the sharp Fermi surface will smear out, allowing states with nonzero Fermi occupations above and below the Fermi surface to contribute to the generation of current. Interestingly, one recent study discovered that the subbandgap photocurrents can be used to probe the magnitude of finite lifetimes [32], in contrast to the "clean limit" and independence of $\tau$ here.

For a general Bloch Hamiltonian $\hat{H}_{0}(\boldsymbol{k})$, in addition to $j^{\text {Fermi }}$ there is another Fermi surface contribution to the current:

$$
\begin{aligned}
\boldsymbol{j}^{\text {Fermi2 }}= & -\sum_{n, i, j, \boldsymbol{k}, \omega^{\prime}= \pm \omega} \frac{e^{3}}{2 V \hbar^{3}}\left\langle u_{n}(\boldsymbol{k})\left|\partial_{k_{i}} \partial_{k_{j}} \hat{H}_{0}(\boldsymbol{k})\right| u_{n}(\boldsymbol{k})\right\rangle \\
& \times \nabla_{\boldsymbol{k}} f_{n}^{T}(\boldsymbol{k}, \mu) A_{i}\left(\omega^{\prime}\right) A_{j}\left(-\omega^{\prime}\right) .
\end{aligned}
$$

Derivation of this contribution is given in the general Bloch Hamiltonian part in Supplemental Material [30]. As shown (see Supplemental Material [30]), the sum of two Fermi surface contributions, Eqs. (6) and (7) is equivalent to the sum of the Drude contribution, Berry curvature dipole contribution and the free-carrier contribution derived in length gauge $[33,34]$. The Drude and Berry curvature dipole contributions can be decomposed from $j^{\text {Fermi2 }}$ but they only represent partial metallic contributions. We note $j^{\text {Fermi2 }}$ vanishes if the energy dispersion relation of $\hat{H}_{0}(\boldsymbol{k})$ is linear or quadratic in the crystal momentum $\boldsymbol{k}$.

By breaking TRS, $\boldsymbol{v}_{n m}(\boldsymbol{k})$ and $\boldsymbol{v}_{n m}(-\boldsymbol{k})$ are no longer negative complex conjugates. BPVE phenomena are enriched in magnetic systems, as $\boldsymbol{j}^{\text {inj }}, \boldsymbol{j}^{\text {shift }}$ and $\boldsymbol{j}^{\text {Fermi }}$ are nonvanishing for driving fields with either LP and CP components. We summarize and classify our results accordingly in Table I.

\section{EXAMPLE: SINGLE WEYL POINT}

We calculate this Fermi surface contribution $\boldsymbol{j}^{\text {Fermi }}$ for $a$ single isotropic Weyl node in a minimal two-band model:

$$
H_{0}=v_{0} \sum_{\alpha=x, y, z} k_{\alpha} \sigma_{\alpha} .
$$

Here $v_{0}$ is the Fermi velocity, and $\sigma_{\alpha}$ are the Pauli matrices. The system is illuminated by CP light described by the vector field $\boldsymbol{A}(t)=A_{0}(\cos (\omega t) \hat{\boldsymbol{x}}+\sin (\omega t) \hat{\boldsymbol{y}})$ that propagates in the $\hat{z}$ direction. With linearly dispersive bands, $\boldsymbol{j}^{\text {Fermi2 }}$ is zero.

At zero temperature, we write $j^{\text {Fermi }}$ in a different form:

$$
\begin{aligned}
\boldsymbol{j}^{\text {Fermi }}= & \frac{e^{3} \omega i}{V \hbar^{2}} \sum_{n, m, i, j, \boldsymbol{k}} \frac{\left(\varepsilon_{n}(\boldsymbol{k})-\varepsilon_{m}(\boldsymbol{k})\right)^{2}}{\left(\varepsilon_{n}(\boldsymbol{k})-\varepsilon_{m}(\boldsymbol{k})\right)^{2}-\hbar^{2} \omega^{2}} \\
& \times \delta\left(\varepsilon_{n}(\boldsymbol{k})-\mu\right) \boldsymbol{v}_{n n}(\boldsymbol{k}) \tilde{\Omega}_{n m}^{i j}(\boldsymbol{k}) A^{i}(\omega) A^{j}(-\omega),
\end{aligned}
$$

where $\tilde{\Omega}_{n m}^{i j}(\boldsymbol{k})=-i\left(R_{n m}^{i}(\boldsymbol{k}) R_{m n}^{j}(\boldsymbol{k})-R_{n m}^{j}(\boldsymbol{k}) R_{m n}^{i}(\boldsymbol{k})\right)$ is the band-resolved Berry curvature. In this way, the DC charge current can be related with the integration of a weighted Berry curvature dipole over the Brillouin zone [27,35,36]. With $\boldsymbol{A}(t)$ introduced above, $\boldsymbol{j}^{\text {Fermi }}$ only has a $\hat{z}$ component,

$$
\begin{aligned}
j^{\text {Fermi }, z}= & \frac{e^{3} i}{V \hbar^{2}} \frac{\omega(2 \mu)^{2}}{(2 \mu)^{2}-\hbar^{2} \omega^{2}} \sum_{\boldsymbol{k}} \delta\left(k-k_{F}\right) \\
& \times \hat{n}^{z}(\boldsymbol{k}) \Omega^{z}(\boldsymbol{k}) A^{x}(\omega) A^{y}(-\omega),
\end{aligned}
$$

where $\hat{n}^{z}(\boldsymbol{k})$ is the $\hat{z}$ component of unit vector normal to the Fermi surface, $\Omega^{z}(\boldsymbol{k})$ is the $\hat{z}$ component of the Berry curvature, and $\mu$ is the chemical potential. For this simple model, $\left|\varepsilon_{n}(\boldsymbol{k})-\varepsilon_{m}(\boldsymbol{k})\right|=2|\mu|$ and is constant across the Fermi surface. For arbitrary CP light illumination, the current can be written as $j^{\text {Fermi, } i}=\sum_{j} \chi_{i j}(\omega)\left[\boldsymbol{E}(\omega) \times \boldsymbol{E}^{*}(\omega)\right]_{j}$, where $\chi_{i j}(\omega)$ is a purely imaginary photovoltaic tensor with the property

$$
\operatorname{Tr}\left[\chi_{i j}(\omega)\right]=i \frac{e^{3}}{h^{2}} \frac{(2 \mu)^{2}}{\omega\left[(2 \mu)^{2}-\hbar^{2} \omega^{2}\right]} Q_{n},
$$

where $Q=\frac{1}{2 \pi} \int_{F S} d \boldsymbol{S} \cdot \boldsymbol{\Omega}(\boldsymbol{k})$ is the charge of the Weyl point. In the limit of $|\hbar \omega| \ll|\mu|$, the current is proportional to the Weyl node's charge, and the trace of $\chi(\omega)$ is proportional to the Chern number of the Fermi surface which encloses 
TABLE I. The classification of all types of contributions to the nonlinear DC photocurrent. "Intrinsic/extrinsic" means whether the current is independent/dependent on $\tau$. "Diagonal/off-diagonal" refers to the part of $\hat{\rho}$ from which the current arises. The last four columns give the correspondence between each current and light polarization with and without TRS. Each contribution is gauge invariant, independent of the choice of phase of the Bloch wave functions.

\begin{tabular}{|c|c|c|c|c|c|c|c|c|}
\hline \multirow[t]{2}{*}{ Contribution } & \multicolumn{2}{|c|}{ Type } & \multicolumn{2}{|c|}{ Origin } & \multicolumn{2}{|c|}{ TR invariant } & \multicolumn{2}{|c|}{ TR broken } \\
\hline & Intrinsic & Extrinsic & Diagonal & Off-diagonal & LP & $\mathrm{CP}$ & LP & $\mathrm{CP}$ \\
\hline Injection & & $\sqrt{ }$ & $\sqrt{ }$ & & & $\sqrt{ }$ & $\sqrt{ }$ & $\sqrt{ }$ \\
\hline Ballistic & & $\sqrt{ }$ & $\sqrt{ }$ & & $\sqrt{ }$ & $\sqrt{ }$ & $\sqrt{ }$ & $\sqrt{ }$ \\
\hline Shift & $\sqrt{ }$ & & & $\sqrt{ }$ & $\sqrt{ }$ & & $\sqrt{ }$ & $\sqrt{ }$ \\
\hline Fermi surface & $\sqrt{ }$ & & $\sqrt{ }$ & $\sqrt{ }$ & & $\sqrt{ }$ & $\sqrt{ }$ & $\sqrt{ }$ \\
\hline Fermi surface2 & $\sqrt{ }$ & & $\sqrt{ }$ & $\sqrt{ }$ & $\sqrt{ }$ & & $\sqrt{ }$ & \\
\hline
\end{tabular}

this topological degeneracy. The charge is a purely topological aspect of the band structure and is robust even when the isotropic symmetry of the Weyl node is broken and the Fermi surface degenerates into an elliptic surface. Consistent with the injection current, the sign of the photocurrent is also dictated by the charge of the Weyl point, and can be used to detect the chirality of the band singularity [37]. We note that for a tilted Weyl cone the energy difference between bands is not constant along the Fermi surface and the $\operatorname{Tr}\left[\chi_{i j}(\omega)\right]$ cannot be reduced to the simple form given in Eq. (11).

Unlike the quantized circular photogalvanic effect [8], here the current does not originate from coherence of optically coupled band states, but instead originates from electrons with crystal momentum along the Fermi surface. In addition, the effect is intrinsic and only depends on the light frequency $\omega$ and not on an extrinsic scattering time $\tau$. This distinguishes itself from the injection current in that due to its coherent nature it does not require a finite $\tau$ to populate carriers [38]. For metallic systems illuminated by midinfrared light, $j^{\text {Fermi }}$ is significant and comparable to $\boldsymbol{j}^{\text {inj }}$. Unlike the difference frequency generation scenario studied in other work [34], $j^{\text {Fermi }}$ can be excited by one monochromatic, polarized light.

\section{EXAMPLE: MULTIPLE WEYL NODES}

We now demonstrate calculation of $j^{\text {Fermi }}$ for a timereversal symmetric, but inversion and mirror broken Weyl semimetal. For systems with TRS, Weyl points in the band structure must come in pairs. The two Weyl points in a pair have the same energy and topological charge, but are located at points in the Brillouin zone with opposite crystal momentum. The topology of the Brillouin zone demands that the sum of the charges of all Weyl points in the Brillouin zone must vanish: $\sum_{n} Q_{n}=0$ forcing the number of Weyl points in a time-reversal symmetric system to be a multiple of four. If mirror symmetry is broken, Weyl points of opposite sign need not occur at the same energy [8], allowing the energy differences between bands, $\Delta(\boldsymbol{k})=\epsilon_{n}(\boldsymbol{k})-\epsilon_{m}(\boldsymbol{k})$, near each Weyl point to be inequivalent (see Fig. 1). We have seen that for a single Weyl point and for light illumination $|\hbar \omega| \ll|\mu|$, the trace of $\chi(\omega)$ is simply proportional to the integral of the Berry curvature across the Fermi surface. For a system of isotropic Weyl points illuminated by light with arbitrary $\omega$, $\Delta(\boldsymbol{k}) \rightarrow \Delta$ and we may write the trace of $\chi(\omega)$ as

$$
\operatorname{Tr}\left[\chi_{i j}(\omega)\right]=i \frac{e^{3}}{h^{2}} \sum_{n} \frac{\left(\Delta_{n}\right)^{2}}{\omega\left[\left(\Delta_{n}\right)^{2}-\hbar^{2} \omega^{2}\right]} Q_{n} .
$$

Here $Q_{n}$ is the charge of node $n$ and $\Delta_{n}$ is the energy difference between Bloch states with crystal momentum along the Fermi surface near Weyl node $n$. With mirror symmetry, $\Delta_{n}$ are the same for differently charged Weyl points. No charge current flows, but only a chiral current represents a charge pumping between Weyl nodes with different chiralities, which is not observable and is similar to the chiral anomaly [39-41]. If $\Delta_{n}$ are different, the trace of $\chi(\omega)$ will be nonzero. Unlike the quantized CPGE induced by injection current where Pauli blocking can forbid the photocurrent when upper state is filled [8], the chemical potential can sit either above or below the Weyl node. Each node's contribution to the current does not change its sign whether the Fermi surface is an electron or hole pocket. In the limit $|\hbar \omega| \ll\left|\Delta_{n}\right|$, we can expand

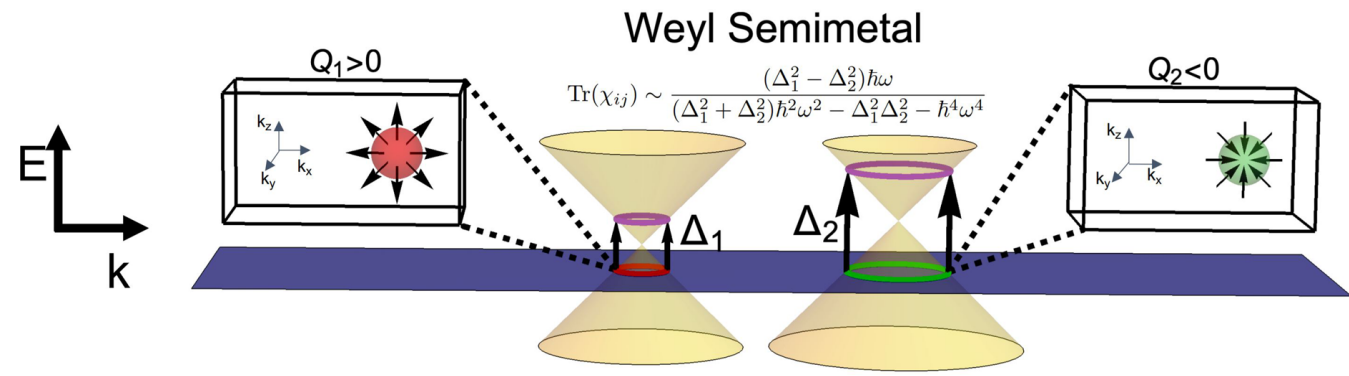

FIG. 1. Illustration of two Weyl points with opposite charge $Q_{n}$ near the Fermi surface. The Fermi surface is shown by the blue plane that intersects Weyl cones at the red and green conic sections. Optical excitations are along the Fermi surface and transition energies $\left(\Delta_{1}\right.$ and $\left.\Delta_{2}\right)$ are different at the two cones. 

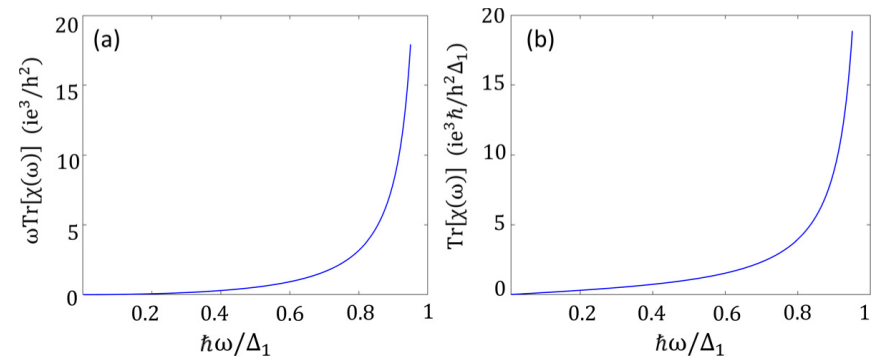

FIG. 2. The relation between $\omega$ and (a) $\omega \operatorname{Tr}[\chi(\omega)]$ (b) $\operatorname{Tr}[\chi(\omega)]$ for a four Weyl nodes system with $Q_{1}=Q_{2}=1, \Delta_{1}=\Delta_{2}$ and $Q_{3}=$ $Q_{4}=-1, \Delta_{3}=\Delta_{4}=2 \Delta_{1}$.

$\operatorname{Tr}\left[\chi_{i j}(\omega)\right]$ in powers of $\hbar^{2} \omega^{2} /\left(\Delta_{n}\right)^{2}$,

$$
\begin{aligned}
\operatorname{Tr}\left[\chi_{i j}(\omega)\right] & \approx i \frac{e^{3}}{\omega h^{2}} \sum_{n}\left(1+\left(\frac{\hbar \omega}{\Delta_{n}}\right)^{2}+\mathcal{O}\left(\frac{\hbar \omega}{\Delta_{n}}\right)^{4}\right) Q_{n} \\
& \approx \frac{i e^{3} \omega}{(2 \pi)^{2}} \sum_{n} \frac{Q_{n}}{\left(\Delta_{n}\right)^{2}}
\end{aligned}
$$

We see that the leading-order term in the expansion is linear in $\omega$, with slope determined by the ratio of the charges of Weyl nodes to the energy differences of the Bloch bands near the Weyl nodes. The relationships between $\omega$ and $\omega \operatorname{Tr}[\chi(\omega)]$, and $\omega$ and $\operatorname{Tr}[\chi(\omega)]$ for a minimal four-Weyl node system are plotted in Fig. 2. Breaking the isotropy of the Weyl nodes takes $\Delta_{n} \rightarrow \Delta_{n}(\boldsymbol{k})$, and the leading contribution to the trace of $\chi(\omega)$ will no longer be directly proportional to the charges of the Weyl nodes. However, the first-order nonvanishing contribution to the current will maintain a linear relationship to the frequency of light.

\section{CANDIDATE MATERIALS}

Noncentrosymmetric metals characterized by the coexistence of metallicity and ferroelectric distortions provide a promising platform for observing this addition to the nonlinear current. Experiments have demonstrated that metallic $\mathrm{LiOsO}_{3}$ and $\mathrm{Cd}_{2} \mathrm{Re}_{2} \mathrm{O}_{7}$ experience a centrosymmetric to noncentrosymmetric phase transition at 140 and at $200 \mathrm{~K}$, respectively $[42,43]$; while the engineering of interfaces in $\mathrm{ANiO}_{3} / \mathrm{LaAlO}_{3}$ heterostructures provides another scheme for achieving other interesting noncentrosymmetric metals [44]. In addition, recent studies on few-layer topological semimetal $\mathrm{WTe}_{2}$ have demonstrated a switchable ferroelectric polarization that could also provide a platform to observe large $j^{\text {Fermi }}$ under illumination by CP light [45].

\section{ACKNOWLEDGMENTS}

L.G. and A.M.R. acknowledge the support of the U.S. Department of Energy, Office of Basic Energy Sciences, under Grant No. DE-FG02-07ER46431. Z.A. and E.J.M. are supported by the U.S. Department of Energy under Grant No. DE-FG02-84ER45118. We acknowledge F. de Juan, T. Morimoto, J.E. Moore and A.G. Grushin for sharing their work with us and pointing out that a similar form of Eq. 6 can also be derived in the length gauge [34]. We also acknowledge H. Watanabe and Y. Yanese for informing us of the update of Ref. [33]. We finally acknowledge D. Kaplan for useful discussion at the finite lifetime limit [32].

L.G. led the analytic derivations and performed all of the numerical calculations. Z.A. participated actively in the derivations and their interpretation. L.G. and Z.A. wrote the manuscript, and E.J.M. and A.M.R. edited the manuscript. E.J.M. and A.M.R. supervised all aspects of the project.
[1] B. I. Sturman and V. M. Fridkin, The Photovoltaic and Photorefractive Effects in Noncentrosymmetric Materials, edited by G. W. Taylor, Ferroelectricity and Related Phenomena, Vol. 8 (Gordon and Breach, London, 1992).

[2] V. Alperovich, V. Belinicher, V. Novikov, and A. Terekhov, Photogalvanic effects investigation in gallium arsenide, Ferroelectrics 45, 1 (1982).

[3] B. I. Sturman, Ballistic and shift currents in the bulk photovoltaic effect theory, Phys. Usp. 63, 407 (2019).

[4] H. T. Duc, C. Ngo, and T. Meier, Ballistic photocurrents in semiconductor quantum wells caused by the excitation of asymmetric excitons, Phys. Rev. B 100, 045308 (2019).

[5] Z. Dai, A. M. Schankler, L. Gao, L. Z. Tan, and A. M. Rappe, Phonon-Sssisted Ballistic Current from First-Principles Calculations,Phys. Rev. Lett. 126, 177403 (2021).

[6] J. E. Sipe and A. I. Shkrebtii, Second-order optical response in semiconductors, Phys. Rev. B 61, 5337 (2000).

[7] N. Laman, M. Bieler, and H. Van Driel, Ultrafast shift and injection currents observed in wurtzite semiconductors via emitted terahertz radiation, J. Appl. Phys. 98, 103507 (2005).

[8] F. de Juan, A. G. Grushin, T. Morimoto, and J. E. Moore, Quantized circular photogalvanic effect in weyl semimetals, Nat. Commun. 8, 15995 (2017).
[9] R. von Baltz and W. Kraut, Theory of the bulk photovoltaic effect in pure crystals, Phys. Rev. B 23, 5590 (1981).

[10] A. M. Burger, R. Agarwal, A. Aprelev, E. Schruba, A. Gutierrez-Perez, V. M. Fridkin, and J. E. Spanier, Direct observation of shift and ballistic photovoltaic currents, Sci. Adv. 5, eaau5588 (2019).

[11] A. M. Burger, L. Gao, R. Agarwal, A. Aprelev, J. E. Spanier, A. M. Rappe, and V. M. Fridkin, Shift photovoltaic current and magnetically induced bulk photocurrent in piezoelectric sillenite crystals, Phys. Rev. B 102, 081113(R) (2020).

[12] S. M. Young and A. M. Rappe, First-Principles Calculation of the Shift Current Photovoltaic Effect in Ferroelectrics, Phys. Rev. Lett. 109, 116601 (2012).

[13] J. Ibañez-Azpiroz, S. S. Tsirkin, and I. Souza, Ab initio calculation of the shift photocurrent by Wannier interpolation, Phys. Rev. B 97, 245143 (2018).

[14] C. Wang, X. Liu, L. Kang, B.-L. Gu, Y. Xu, and W. Duan, Firstprinciples calculation of nonlinear optical responses by wannier interpolation, Phys. Rev. B 96, 115147 (2017).

[15] L. Z. Tan and A. M. Rappe, Enhancement of the Bulk Photovoltaic Effect in Topological Insulators, Phys. Rev. Lett. 116, 237402 (2016). 
[16] K. W. Kim, T. Morimoto, and N. Nagaosa, Shift charge and spin photocurrents in Dirac surface states of topological insulator, Phys. Rev. B 95, 035134 (2017).

[17] Y. Zhang, H. Ishizuka, J. van den Brink, C. Felser, B. Yan, and N. Nagaosa, Photogalvanic effect in weyl semimetals from first principles, Phys. Rev. B 97, 241118(R) (2018).

[18] Z. Li, T. Iitaka, H. Zeng, and H. Su, Optical response of the chiral topological semimetal rhsi, Phys. Rev. B 100, 155201 (2019).

[19] A. M. Cook, B. M. Fregoso, F. De Juan, S. Coh, and J. E. Moore, Design principles for shift current photovoltaics, Nat. Commun. 8, 14176 (2017).

[20] T. Rangel, B. M. Fregoso, B. S. Mendoza, T. Morimoto, J. E. Moore, and J. B. Neaton, Large Bulk Photovoltaic Effect and Spontaneous Polarization of Single-Layer Monochalcogenides, Phys. Rev. Lett. 119, 067402 (2017).

[21] Y. Zhang, T. Ideue, M. Onga, F. Qin, R. Suzuki, A. Zak, R. Tenne, J. Smet, and Y. Iwasa, Enhanced intrinsic photovoltaic effect in tungsten disulfide nanotubes, Nature (London) 570, 349 (2019).

[22] D. J. Passos, G. B. Ventura, J. M. Viana Parente Lopes, J. M. B. L. dos Santos, and N. M. R. Peres, Nonlinear optical responses of crystalline systems: Results from a velocity gauge analysis, Phys. Rev. B 97, 235446 (2018).

[23] J. E. Sipe and E. Ghahramani, Nonlinear optical response of semiconductors in the independent-particle approximation, Phys. Rev. B 48, 11705 (1993).

[24] G. B. Ventura, D. J. Passos, J. M. B. Lopes dos Santos, J. M. Viana Parente Lopes, and N. M. R. Peres, Gauge covariances and nonlinear optical responses, Phys. Rev. B 96, 035431 (2017).

[25] K. Rzazewski and R. W. Boyd, Equivalence of interaction Hamiltonians in the electric dipole approximation, J. Mod. Opt. 51, 1137 (2004).

[26] V. Genkin and P. Mednis, Contribution to the theory of nonlinear effects in crystals with account taken of partially filled bands, Sov. Phys. JETP 27, 609 (1968).

[27] O. Matsyshyn and I. Sodemann, Nonlinear Hall Acceleration and the Quantum Rectification Sum Rule, Phys. Rev. Lett. 123, 246602 (2019).

[28] J. Provost and G. Vallee, Riemannian structure on manifolds of quantum states, Commun. Math. Phys. 76, 289 (1980).

[29] M. V. Berry, Quantal phase factors accompanying adiabatic changes, Proc. R. Soc. London A 392, 45 (1984).

[30] See Supplemental Material at http://link.aps.org/supplemental/ 10.1103/PhysRevResearch.3.L042032 for the full derivation at infinite band limit and general Bloch Hamiltonian, the equivalence between velocity gauge and length gauge, and the analytical calculations for model Hamiltonians.
[31] J. Von Neumann, Wahrscheinlichkeitstheoretischer aufbau der quantenmechanik, Nachr. Ges. Wiss. Goettingen, Math. Phys. K1. 1927, 245 (1927).

[32] D. Kaplan, T. Holder, and B. Yan, Nonvanishing Subgap Photocurrent as A Probe of Lifetime Effects, Phys. Rev. Lett. 125, 227401 (2020).

[33] H. Watanabe and Y. Yanase, Chiral Photocurrent in ParityViolating Magnet and Enhanced Response in Topological Antiferromagnet, Phys. Rev. X 11, 011001 (2021).

[34] F. de Juan, Y. Zhang, T. Morimoto, Y. Sun, J. E. Moore, and A. G. Grushin, Difference frequency generation in topological semimetals, Phys. Rev. Research 2, 012017(R) (2020).

[35] I. Sodemann and L. Fu, Quantum Nonlinear Hall Effect Induced by Berry Curvature Dipole in Time-Reversal Invariant Materials, Phys. Rev. Lett. 115, 216806 (2015).

[36] H. Rostami and M. Polini, Nonlinear anomalous photocurrents in weyl semimetals, Phys. Rev. B 97, 195151 (2018).

[37] Q. Ma, S.-Y. Xu, C.-K. Chan, C.-L. Zhang, G. Chang, Y. Lin, W. Xie, T. Palacios, H. Lin, S. Jia et al., Direct optical detection of weyl fermion chirality in a topological semimetal, Nat. Phys. 13, 842 (2017).

[38] J. Ma, Q. Gu, Y. Liu, J. Lai, P. Yu, X. Zhuo, Z. Liu, J.-H. Chen, J. Feng, and D. Sun, Nonlinear photoresponse of type-ii weyl semimetals, Nat. Mater. 18, 476 (2019).

[39] A. A. Zyuzin and A. A. Burkov, Topological response in weyl semimetals and the chiral anomaly, Phys. Rev. B 86, 115133 (2012).

[40] A. Burkov, Chiral anomaly and transport in weyl metals, J. Phys.: Condens. Matter 27, 113201 (2015).

[41] A. Burkov, Weyl metals, Annu. Rev. Condens. Matter Phys. 9 , 359 (2018).

[42] I. A. Sergienko, V. Keppens, M. McGuire, R. Jin, J. He, S. H Curnoe, B. C. Sales, P. Blaha, D. J. Singh, K. Schwarz, and D. Mandrus et al., Metallic "Ferroelectricity" in the Pyrochlore Cd2Re2O7, Phys. Rev. Lett. 92, 065501 (2004).

[43] Y. Shi, Y. Guo, X. Wang, A. J. Princep, D. Khalyavin, P. Manuel, Y. Michiue, A. Sato, K. Tsuda, S. Yu et al., A ferroelectric-like structural transition in a metal, Nat. Mater. 12, 1024 (2013).

[44] T. Kim, D. Puggioni, Y. Yuan, L. Xie, H. Zhou, N. Campbell, P. Ryan, Y. Choi, J.-W. Kim, J. Patzner et al., Polar metals by geometric design, Nature (London) 533, 68 (2016).

[45] Z. Fei, W. Zhao, T. A. Palomaki, B. Sun, M. K. Miller, Z. Zhao, J. Yan, X. Xu, and D. H. Cobden, Ferroelectric switching of a two-dimensional metal, Nature (London) 560, 336 (2018).

Correction: The omission of a "Corresponding author" identifier and email address has been fixed. 